\title{
Influence of an inductive impedance of the time evolution of the distribution function ${ }^{1}$
}

\author{
H. Moshammer \\ Stanford Linear Accelerator Center, \\ Stanford University, Stanford, CA 94309 \\ SLAC/AP-94 \\ DE93 006914
}

\section{Motivation}

Suppose a beam, whose distribution is known, is injected into a transport line (storage ring or linac), and let us assume that the beam is not matched to this transport line. As a consequence, the beam distribution will rotate in phase space. Furthermore, linear and nonlinear perturbation will start to deform the geometrical shape of the incoming beam distribution. The result of this dilution process is an apparently bigger beam emittance at the end of the transport line.

The impact of the nonlinear RF field on the injected beam has been studied in Ref. [1]. The turn-by-turn accumulative effect can be described by averaging the nonlinear field terms in the Hamiltonian. Figure 1 illustrates this method by comparing the analytically-derived distribution function to multi-particle tracking.

multi particle simulation

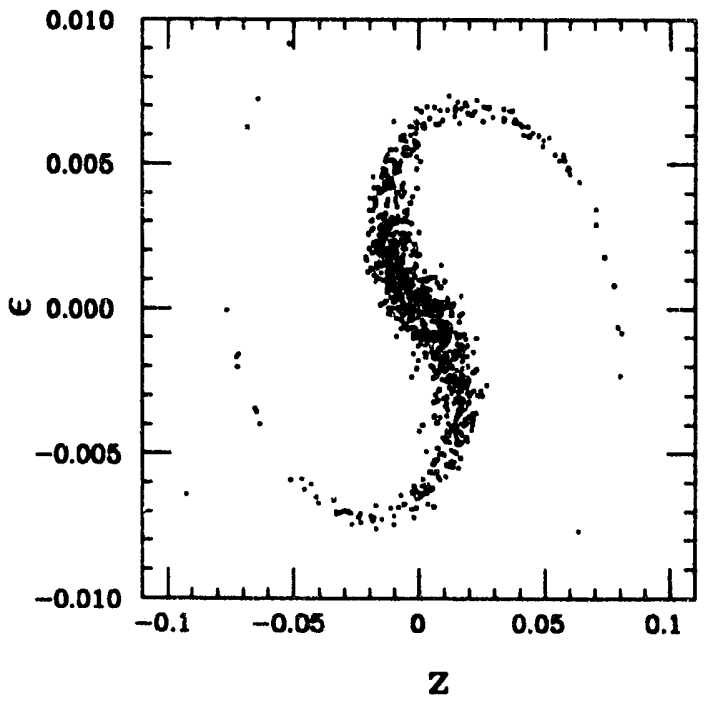

distribution function

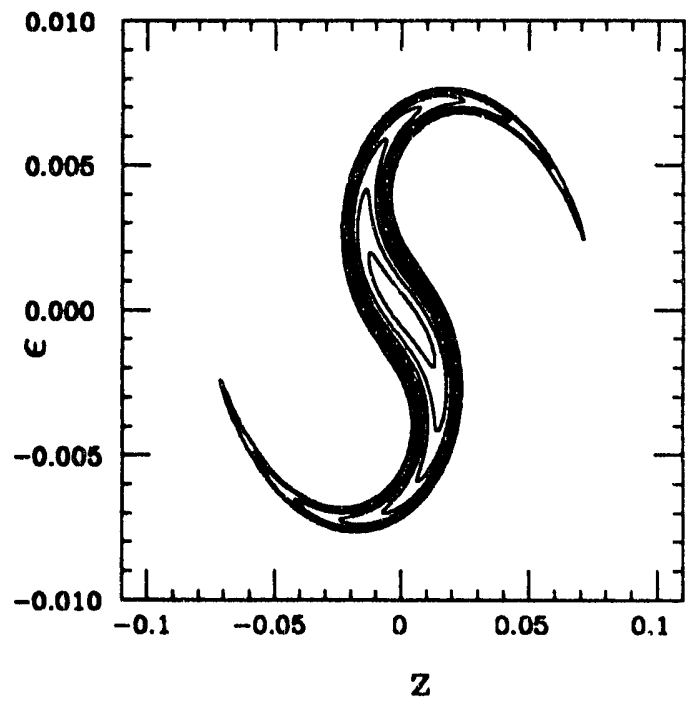

Figure 1. Effect of the nonlinear RF field on a mismatched injected beam.

The evolution of the distribution function in analytic form contains much more than a good visual agreement between the analytically-derived and the tracked distribution in phase space.

1. Transverse matching of transport lines: A parameter composed of the $\beta$ functions of the beam and the $\beta$ functions of the lattice may be identified as a driving term of the filamentation process. Assuming an octupole-like perturbation in a storage ring (or a chromatic perturbation in a linac), this parameter is the well-known $\beta_{\text {mag }}$ factor [2].

\footnotetext{
${ }^{1}$ Work supported by the Department of Energy, contract DE-AC03-76SF00515.
} 
2. First and second moments may be derived from the relatively simple analytic expression of the distribution function. A comparison between actual measurements of the center of mass and beam size and these analytic expressions may allow the injection to be optimized. The expression of the first moment contains the information of the incoming beam emittance and may be used to extract the beam emittance out of turn-by-turn BPM data.

In this paper we want to treat the influence of an inductive wakefield on the time evolution of the distribution function. To be specific, we work in the longitudinal phase space and assume the transport line to be a storage ring.

\section{The Hamiltonian}

I must say first that this work should be primarily regarded as a proof of principle. The application to a more realistic wakefield model is reserved for future studies. From this point of view, the results obtained here are not directly applicable.

We use the canonical coordinate $\epsilon=\Delta E / E$ to describe the relative energy deviation, and the canonical momentum $z$ which denotes the longitudinal position of a particle in the bunch. The change in energy and in longitudinal position over one revolution $2 R \pi$ is given by

$$
\begin{gathered}
\epsilon^{\prime}=\frac{1}{2 R \pi E_{0}}\left(-e V_{R F}(z)+U_{\text {rad }}+e V_{i n d}(z)\right), \\
z^{\prime}=-\alpha \epsilon
\end{gathered}
$$

where $\alpha$ denotes the momentum compaction factor. For the accelerating RF we use $V_{R F}=\hat{V} \sin \left(\phi_{s}+\Delta \phi\right)$. The difference in phase and the longitudinal position are related by the harmonic number $\Delta \phi=h z / R$. With the usual definition of the synchrotron tune $\nu_{s}$ and a Taylor expansion of the RF field, we obtain the Hamiltonian

$$
H(\epsilon, z)=\frac{1}{2} \alpha \epsilon^{2}+\frac{\nu_{s}^{2}}{\alpha R^{2}}\left(\frac{1}{2} z^{2}-\tan \left(\phi_{s}\right) \frac{h}{R} \frac{z^{3}}{3 !}-\frac{h^{2}}{R^{2}} \frac{z^{4}}{4 !}-\frac{1}{V_{0}^{\prime \prime}} \int_{0}^{z} V_{\text {ind }}(\zeta) d \zeta\right)+O\left(z^{5}\right),
$$

where we used $V_{0}^{\prime}=d V_{R F} /\left.d s\right|_{z=0}$. We should keep in mind that the linear term $V(z)=$ const together with the radiation loss $U_{\text {rad }}$ has already been taken care of by $\left.V_{R F}\right|_{z=0}$. At this point we change the canonical coordinates and the independent variable form $(\epsilon, z, s)$ to $(\xi, \eta, t)$ :

$$
\begin{aligned}
& \xi=\epsilon \sqrt{\frac{\alpha R}{\nu_{s}}}=\epsilon \sqrt{\frac{\sigma_{z \infty}}{\sigma_{e \infty}}} \\
& \eta=z \sqrt{\frac{\nu_{s}}{\alpha R}}=z \sqrt{\frac{\sigma_{e \infty}}{\sigma_{z \infty}}},
\end{aligned}
$$

and the new Hamiltonian becomes

$$
\begin{gathered}
H(\xi, \eta, t)=h_{0}(\xi, \eta)+h_{n}(\eta)+h_{w}(\eta, t) \\
h_{0}=\omega_{s}\left(\frac{1}{2} \xi^{2}+\frac{1}{2} \eta^{2}\right), \quad h_{n}=-\omega_{s}\left(\tan \left(\phi_{s}\right) \frac{h}{R} \sqrt{\frac{\alpha R}{\nu_{s}}} \frac{\eta^{3}}{3 !}+\frac{h^{2}}{R} \frac{\alpha}{\nu_{s}} \frac{\eta^{4}}{4 !}\right), \quad h_{w}=-\frac{\omega_{s}}{V_{0}^{\prime}} \int_{0}^{\eta} V_{\text {ind }}(\tilde{\eta}, t) d \tilde{\eta},
\end{gathered}
$$

where $V_{0}^{\prime}=d V_{R F} /\left.d \eta\right|_{\eta=0}$. The bunchlength after mismatched injection oscillates and causes the explicit time dependance in $h_{w}$. Now we simplify and restrict the model to a purely inductive impedance. The induced voltage is given by [3]

$$
V_{\text {ind }}=-L \frac{d I}{d t}
$$


and $h_{w}$ will be proportional to the current $I$. We denote the phase space distribution function by $\Psi$ and may express the current by: $I \sim \int \Psi d \xi$. Thus the Hamiltonian becomes

$$
h_{w}=\frac{\omega_{s} N e c \nu_{s}}{\alpha V_{0}^{\prime}}|Z(n) / n|_{e f f} \int_{-\infty}^{\infty} \Psi(\tilde{\xi}, \eta, t) d \tilde{\xi},
$$

where inductance has been replaced by the impedance: $L=\frac{R}{c}|Z(n) / n|_{\text {eff }}$. We simplify the problem further and introduce a Gaussian unperturbed distribution function into $h_{w} . \Psi_{0}$ is a solution to the Liociville equation

$$
\frac{\partial \Psi}{\partial t}-\frac{\partial h_{0}}{\partial \xi} \frac{\partial \Psi}{\partial \eta}+\frac{\partial h_{0}}{\partial \eta} \frac{\partial \Psi}{\partial \xi}=0
$$

To derive $\Psi_{0}$ we assume the bunch is injected upright into phase space. We denote the bunch length at injection by $\sigma_{z 0}$ and the energy spread at injection by $\sigma_{e 0}$. Then the unperturbed distribution function is given by [1]

$$
\Psi_{0}(\xi, \eta, t)=\frac{1}{2 \pi \sigma} \exp \left(-\frac{b}{2 \sigma}\left(\eta^{2}+\xi^{2}\right)-\frac{c}{2 \sigma}\left(\eta^{2}-\xi^{2}\right) \cos \left(2 \omega_{s} t\right)-\frac{c}{\sigma} \eta \xi \sin \left(2 \omega_{s} t\right)\right)
$$

where $\sigma=\sigma_{z 0} \sigma_{e 0}$ is related to the injected emittance. The other parameters are

$$
b=\frac{1+q^{2}}{2 q}, \quad c=\frac{1-q^{2}}{2 q} \text { and } \quad q=\frac{\sigma_{z 0} \sigma_{e \infty}}{\sigma_{z \infty} \sigma_{e 0}} .
$$

With $q=1$, the distribution function loses its explicit time dependence and the beam is matched. For the SLC damping rings we have $q \approx 1 / 20<1$. The projection of the distribution function on the $\eta$ axis becomes:

$$
\int \Psi_{0} d \xi=\frac{1}{\sqrt{2 \pi \sigma \kappa\left(\omega_{s} t\right)}} \exp \left(-\frac{\eta^{2}}{2 \sigma \kappa\left(\omega_{s} t\right)}\right) \quad \text { with } \quad \kappa\left(\omega_{s} t\right)=b-c \cos \left(2 \omega_{s} t\right)^{6}
$$

With Eq. 7 the Hamiltonian is given by:

$$
h_{w}=\frac{\omega_{s} N e c \nu_{s}}{\alpha V_{0}^{\prime} \sqrt{2 \pi \sigma \kappa\left(\omega_{s} t\right)}}|Z(n) / n|_{e f f} \exp \left(-\frac{\eta^{2}}{2 \sigma \kappa\left(\omega_{s} t\right)}\right) .
$$

The function $k\left(\omega_{s} t\right)$ describes the square of the bunch length.

\section{Averaging of Perturbations}

The averaging method has been applied to beam dynamics problems by various authors in the past. Schematically it may be said that the separation between solutions of perturbed and averaged systems will be small if the exact trajectory spends most of its time in regions where the unperturbed trajectory is ergodic, hence where the "ergodization" of the perturbation (the approximation of the spatial average by a time average along a given trajectory) takes place rapidly. It is worthwhile to examine the role of ergodicity in this method from a general standpoint.

There is first of all the question how rapidly ergodization takes place? We assume that the unperturbed trajectory can be described by conditionally-periodic motion $\boldsymbol{\Phi}, \mathbf{I}=\left(\Phi_{1} \cdots \Phi_{n}, I_{1} \cdots I_{n}\right)$ with $(\dot{\boldsymbol{\Phi}}, \dot{\mathbf{I}})=(\Omega t, 0)$ where $\boldsymbol{\Omega}=$ const. The associated unperturbed Hamiltonian might look like $H_{0}=\Omega I$. The spatial average is defined as [5]

$$
\langle f\rangle_{\Phi}=\frac{1}{(2 \pi)^{n}} \int_{0}^{2 \pi} \cdots \int_{0}^{2 \pi} f(\Phi) d \Phi_{1} \cdots d \Phi_{n}
$$

and the time average as

$$
\langle f\rangle_{T}=\frac{1}{T} \int_{0}^{T} f\left(\Phi_{0}+\Omega t\right) d t
$$


Theorem: $\lim _{T \rightarrow \infty}\langle f\rangle_{T}$ coincides with : e space average if the function $f$ is continuous and the frequencies $\omega_{i}$ are independent, i.e., the relation $\sum_{i=1}^{n} k_{i} \Omega_{i}=0$ implies $n_{i}=0$ [5].

The ergodization of a give $:$ ? function in phase space $\Gamma$ may be realized slowly or rapidly depending on the initial condition of the trajectory $\left(\mathbf{I}_{\mathbf{0}}, \mathbf{\Phi}_{\mathbf{0}}\right)$. From Ref. [6] we use the followirg definition: $\mathbf{E}$ is a certain subset of $\Gamma$ related with the ergodization for the unperturbed motion of a given finction $f$ on $\Gamma$. For $\left(\mathbf{I}_{0}, \Phi_{0}\right) \in$ $\mathbf{E}\left(T_{0}, \delta\right) \subset \boldsymbol{\Gamma}$ and for all $T>T_{0}$

$$
\left\langle\langle f\rangle_{\boldsymbol{\Phi}}-\langle f\rangle_{T}\right|<\delta
$$

the time average of $f$ differs by less than $\delta$ from the spatial average of $f$. For our purposes it seems more appropriate to normalize the difference between time and spatial average. For example, we may use:

$$
\frac{\left|\langle f\rangle_{\Phi}-\langle f\rangle_{T}\right|}{\left|\langle f\rangle_{\boldsymbol{\Phi}}\right|+\left|\langle f\rangle_{T}\right|}<\delta
$$

Since the initial conditions of the trajectories are defined by the distribution function at $t=0$ we may evaluate $r$ : the fraction of all particles which at $t=0$ are located in $\mathbf{E}\left(T_{0}, \delta\right)$. In other words, for a certain fraction of the total number of injected particles denoted by $r$, the ergodization is realized to $\delta$ within $T_{0}$.

In what follows we will illustrate these definitions by four examples which are related to the Hamiltonian derived in Sec. 1. To be specific we choose $T_{0}$ equal one synchrotron period and $\delta=0.1$

Example A: Nonlinear RF Field Averaged over the Unperturbed Motion

Here we focus on the first two terms of Eq. 6. The Hamiltonian will no lonen: aplicitly depand on time

$$
\begin{gathered}
H(\xi, \eta)=h_{0}(\xi, \eta)+h_{n}(\eta) \\
h_{0}=\omega_{s}\left(\frac{1}{2} \xi^{2}+\frac{1}{2} \eta^{2}\right), \quad h_{n}=-\omega_{s} \frac{h^{2} \alpha}{R} \frac{\eta_{s}^{4}}{4 !} .
\end{gathered}
$$

For the sake of simplicity we have neglected the $\eta^{3}$ term. We introduce action angle variablos

$$
\eta=\sqrt{2 I} \cos (\phi), \quad \xi=\sqrt{2 I} \sin (\phi),
$$

and obtain for the spatial average :

$$
\left\langle h_{n}\right\rangle_{\phi}=-\omega_{s} \frac{h^{2} \alpha}{R \nu_{s}} \frac{I^{2}}{16}
$$

For $T>T_{0}=2 \pi / \omega_{s}$ the normalized difference between spatial and time average defined in Lq. 12 becomes:

$$
\frac{\left|\left\langle h_{n}\right\rangle_{\phi}-\left\langle h_{n}\right\rangle_{T}\right|}{\left|\left\langle h_{n}\right\rangle_{\phi}\right|+\left|\left\langle h_{n}\right\rangle_{T}\right|}<\frac{1}{3 \omega_{s} T / 3-1}<0.1
$$

where the estimate is not optimal. For all possible initial conditions $\left(I_{0}, \phi_{0}\right) \in \Gamma$ and $T>2 \pi / \omega_{s}$ the ergodization of $h_{n}$ over the unperturbed trajectory is realized to within $h=0.1$. This statement concerns $100 \%$ of the injected particles, and thus $r=1$.

Example B: Wakefield Averaged over the Unperturbed Motion

Here we select the first and the third term in Eq. 6. The Hamiltonian will depend explicitly on time. With Eq. 11 we have

$$
\begin{gathered}
H(\xi, \eta, t)=h_{0}(\xi, \eta)+h_{w}(\eta, t) \\
h_{0}=\omega_{s}\left(\frac{1}{2} \xi^{2}+\frac{1}{2} \eta^{2}\right), \quad h_{w}=\frac{\omega_{s} \epsilon}{\sqrt{r_{i}}} \exp \left(-\frac{\eta^{2}}{2 \sigma \kappa}\right) \quad \text { with } \quad \epsilon=\frac{N \epsilon c \nu_{s}}{\alpha V_{0}^{\prime} \sqrt{2 \pi \sigma}}|Z(n) / n|_{\text {eff }} .
\end{gathered}
$$


We introduce action angle variables for the unperturbed motion $\left(I_{1}, \Phi_{1}\right)$ given by Eq. 13. The explicit time dependence will be eliminated by introducing a second pair of canonically-conjugate variables $\left(I_{2}, \Phi_{2}=\omega_{s} t\right)$ [4]. It is worth to mention that this second phase is not at all artificial. One might think about $\bar{\Phi}_{2}$ as a coherent phase since it is related to bunch length oscillations, whereas $\Phi_{1}$ describes the incoherent motion. We obtain for the Hamiltonian:

$$
H(\mathbf{I}, \mathbf{\Phi})=\omega_{s} I_{1}+\omega_{s} I_{2}+\frac{\omega_{s} \epsilon}{\sqrt{\kappa\left(\Phi_{2}\right)}} \exp \left(-\frac{I_{1} \cos \left(\Phi_{1}\right)^{2}}{\sigma \kappa\left(\Phi_{2}\right)}\right)
$$

At this point it becomes obvious that this example is a rather pathologic one, since the two frequencies are not independent. Nevertheless, we proceed to evaluate the spatial average. The integration over the $\Phi_{1}$ may be expressed by a Bessel function $J_{0}$

$$
\left\langle h_{w}\right\rangle_{\Phi}=\frac{1}{2 \pi} \int_{0}^{2 \pi} \frac{1}{\sqrt{\kappa\left(\Phi_{2}\right)}} \exp \left(-\frac{I_{1}}{2 \sigma \kappa\left(\Phi_{2}\right)}\right) J_{0}\left(\frac{i I_{1}}{2 \sigma \kappa\left(\Phi_{2}\right)}\right) d \Phi_{2} .
$$

Figure 2 shows the injected beam ellipse in $(z, \epsilon)$ space which contains $97.7 \%$ of all particles ( $2 \sigma$ of a Gaussian distribution). The region of initial conditions $\left(I_{1}, \Phi_{1}\right)_{t=0}$ denoted by $\mathbf{E}$ in which the ergodization is realized to within $\delta=1 / 10$ is shown as the shaded area in Fig. 2. But only $39 \%$ of the injected particles may be found in the shaded region of Fig. 2. Hence, we should not use the trajectory of the unperturbed motion to average over the wakefield contribution.

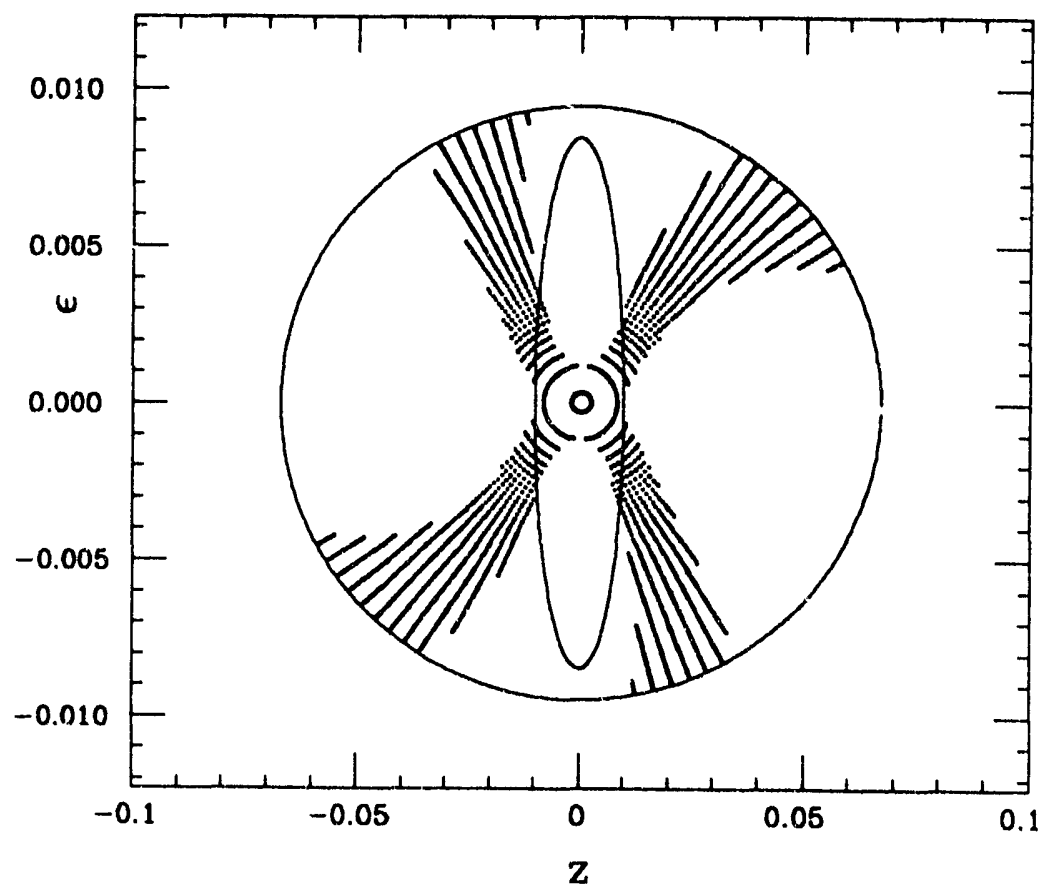

Figure 2. Regions of slow and fast ergodization in Example B according to Eq. 12.

\section{Example C: Nonlinear Part of the Wakefield Averaged over the Linear Motion}

As in Example B we select the first and the third term in Eq. 6. But we split the Hamiltonian in two parts: $h_{l}$ contains all terms that are quadratic in the canonical variables.

$$
\begin{gathered}
H(\xi, \eta, t)=h_{l}(\xi, \eta, t)+h_{n w}(\eta, t) \\
h_{l}=\frac{\omega_{s}}{2}\left(\xi^{2}+\eta^{2}\left(1-\frac{\epsilon}{\sigma \sqrt{\kappa^{3}}}\right)\right), \quad h_{n w}=\frac{\omega_{s} \epsilon}{\sqrt{\kappa}}\left(\exp \left(-\frac{\eta^{2}}{2 \sigma \kappa}\right)+\frac{\eta^{2}}{2 \sigma \kappa}\right)
\end{gathered}
$$


Hamilton's equation with $h_{l}$ leads to a Hill's equation, i.e., linear equation with periodic coefficients and without first derivative terms

$$
\ddot{\eta}+\left(1-\frac{\epsilon}{\sigma \sqrt{\kappa\left(\omega_{s} t\right)^{3}}}\right) \eta=0,
$$

where $\dot{\eta}$ denotes the differentiation w.r.t. $\omega_{s} t$. We introduce action angle variables which are similar to the treatment of the betatron motion [7]

$$
\eta=\sqrt{2 I} w \cos (\Phi) \quad, \quad \xi=\sqrt{2 I}\left(\frac{1}{w} \sin (\Phi)-\dot{w} \cos (\Phi)\right)
$$

where the "amplitude-function" $w$ is a periodic solution of the equation:

$$
\ddot{w}+\left(1-\frac{\epsilon}{\sigma \sqrt{\kappa^{3}}}\right) w=\frac{1}{w^{3}} .
$$

We introduce Eq. 16 into the Hamiltonian and replace $\omega_{s} t$ by a second angle variable $\Phi_{2}$ to eliminate the time dependence

$$
H=\omega_{s} \frac{I_{1}}{w^{2}}+\omega_{s} I_{2}+h_{n w}(\mathbf{I}, \Phi), \quad h_{n w}=\frac{\omega_{s} \epsilon}{\sqrt{\kappa}}\left(\exp \left(-\frac{I_{1} w^{2} \cos \left(\Phi_{1}\right)^{2}}{\sigma \kappa\left(\Phi_{2}\right)}\right)+\frac{I_{1} w^{2} \cos \left(\Phi_{1}\right)^{2}}{\sigma \kappa\left(\Phi_{2}\right)}\right) .
$$

We proceed to evaluate the regions of fast and slow ergodization according to Eq.12 for $\delta=0.1$ and $T_{0}=2 \pi / \omega_{s}$. Only initial conditions which are inside the large ellipse in Fig. 3 have been tested for the criterion Eq. 12. The small ellipse shows the contour of the injected beam containing $97.7 \%$ of all particles. For initial conditions out of the dotted area in Fig. 3 the normalized difference between time and spatial average differs by less than $\delta=1 / 10$. About $94 \%$ of the injected particles occupy this region at injection.

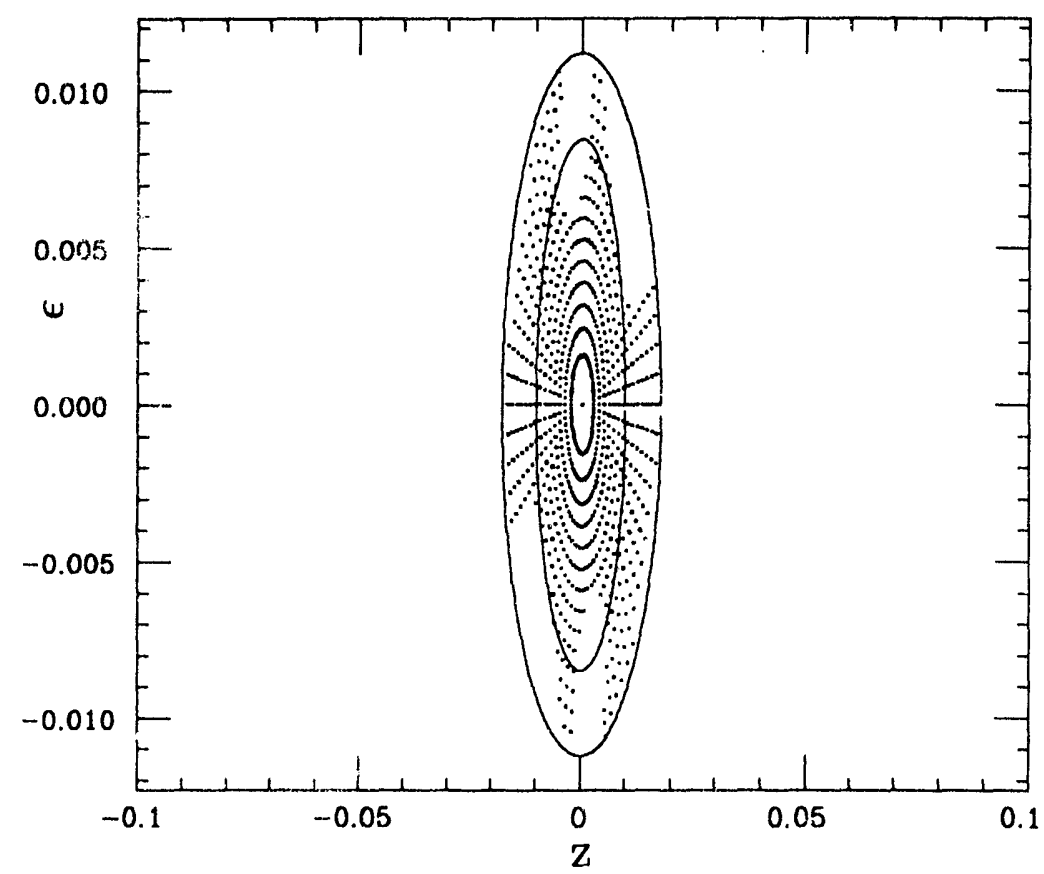

Figure 3. Regions of slow and fast ergodization in Example C according to Eq. 12.

Example D: Nonlinear Part of the RF Averaged over the Linear Motion

We would appreciate it if the linear motion based on Hill's equation would also be able to ergodize the nonlinear terms from the RF. The Hamiltonian we are presently concerned with is:

$$
H(\xi, \eta, t)=h_{l}(\xi, \eta, t)+h_{n}(\eta)
$$


with $h_{l}$ from example 3 and

$$
h_{n}=-\omega_{s} \frac{h^{2} \alpha \eta^{4}}{R \nu_{s} 4 !}=-\omega_{s} \frac{h^{2} \alpha}{R \nu_{s} 3 !} I^{2} w^{4} \cos (\Phi)^{4},
$$

where we used the action angle variables $I, \Phi$ introduced for $h_{l}$ in the previous example. The spatial and the time average becomes:

$\left\langle h_{n}\right\rangle_{\Phi}=\frac{3 \omega_{s}}{16 \pi} \bar{c} I^{2} \int_{0}^{2 \pi / \omega_{s}} w^{4} d t, \quad\left\langle h_{n}\right\rangle_{T}=\frac{1}{T} \bar{c} I^{2} \int_{0}^{T} w^{4} \cos \left(\Phi_{0}+\omega_{s} t\right)^{4} d t$ with $\bar{c}=-\omega_{s} \frac{h^{2} \alpha}{R \nu_{s} 3 !}, T=2 \pi / \omega_{s}$

The normalized difference between the spatial and the time average of $h_{n}$ does not get below $\delta=1 / 10$. Thus $r=0$ and we should not use the trajectory of Hill's equation to average over the nonlinear RF terms.

The consequence of this result is that we have to average the nonlinear wakeforce using Hill's equation and the nonlinear RF force using the linear unperturbed equation according to Example A.

\section{Analysis of the Linear Motion}

We decompose the contribution of the wakefield into a linear part (quadratic in the Hamiltonian) and a nonlinear part. Hamilton's equation based on

$$
h_{l}=\frac{\omega_{s}}{2}\left(\xi^{2}+\eta^{2}\left(1-\frac{\epsilon}{\sigma \sqrt{\kappa^{3}}}\right)\right)
$$

leads to a Hill's equation comparable with the radial displacement in an circular accelerator being focussed and defocussed by quadrupoles. There are two important consequences:

1. The stability of the linear motion depends on the trace of the one-turn map.

2. The amplitude function $w(t)$, which is a periodic solution of Eq. 17, leads to modulations of the shape of the beam ellipse in phase space. This modulation corresponds to the transverse beam size which depends on the product of the emittance and the $\beta$-function.

From the one-turn map $M$ in Eq. 15 we obtain the amplitude function at $t=0: w_{0}, \dot{w}_{0}$ following the procedure in Ref. [8]. The trace of $M$ answers the stability of the linear motion. It depends, via $\epsilon$, on the impedance $|Z(n) / n|_{\text {eff }}$ and on the number of injected particles, but also on the quantity $q$ that was introduced in Eq. 10. We keep in mind that with $q=1$ the injected beam ellipsoid degenerates into a circle and the Hamiltonian in Eq. 19 loses its explicit time dependence.

We use a Runge-Kutta integrator to obtain the one-turn map $M$ from Eq. 15. The eigenvalues of the linear map are given by [8]:

$$
\lambda^{2}-\operatorname{Tr}(M) \lambda+1=0 .
$$

If $\lambda$ is an eigenvalue then $1 / \lambda$ is also an eigenvalue. If $|\operatorname{Tr}(M)|>2$ the eigenvalues of $M$ do not lie on the unit cycle in the complex plane and the motion becomes unstable according to the stability definition in Ref. [5]:

Definition A transformation $M$ is called stable if for all $\epsilon>0$ there exists a $\delta>0$ such that

$$
|\mathbf{x}|<\delta \Rightarrow\left|M^{n} \mathbf{x}\right|<\epsilon .
$$

A useful quantity is $\tau$, the number of synchrotron periods necessary to increase the second moment of the initial distribution by a factor of two

$$
\left\langle x_{i}^{2}\right\rangle_{t=\tau}=\lambda^{2 \tau / T_{0}}\left\langle x_{i}^{2}\right\rangle_{t=0}=2\left\langle x_{i}^{2}\right\rangle_{t=0} \quad \rightarrow \quad \tau=\frac{1}{2} T_{0} \frac{\ln (2)}{\ln \lambda} .
$$


Here $x_{i}$ corresponds to the eigenvector with the eigenvalue $|\lambda|>1$. In Fig. 4a we show the stability diagram of Eq. 15 in the $\left(N, b=\left(q^{2}+1\right) / 2 q\right)$ parameter space. In the first unstable region we evaluated the contour lines of the growth rates $\tau=1 / 2,1 / 4$. The parameters are chosen as $q=1 / 6-1 / 60,|Z(n) / n|_{\text {eff }}=2.6, \sigma_{z 0}=$ $5-0.5[\mathrm{~mm}], \sigma_{e 0}=0.42 \%$.

What happens if we inject under unstable conditions? Notice that the wakeforce decreases as we go to higher amplitude due to the contribution of $h_{n w}$ in Eq. 18. Particles with small initial amplitude will not be lost but will drift to regions of higher amplitude where the wakeforce is weaker. Thus we might find a depletion of particles of small amplitude.

The three crosses in Fig. $4 a$ at $b=10$ correspond to stable and unstable linear motion. We illustrate these three injection conditions with multi-particle tracking where the nonlinear wakefield has been included with Eq. 11. About 25, '00 particles have been tracked for 100 and 500 revolutions which corresponds to 1.16 and 5.82 synchrotron pe $1, d \mathrm{ds}$. In Fig. $4 \mathrm{~b}$ we plot the projection of the distribution function on the $z$ axis for $N=4.5,7.5$ and $1210^{10}$ injected particles. One clearly observes a drift of particles with small amplitude to higher amplitudes for $N=7.510^{10}$ which lies in the region of unstable linear motion. The presence of the nonlinear wakefield term causes a diffusion which counteracts the depletion process in regions of small amplitudes.

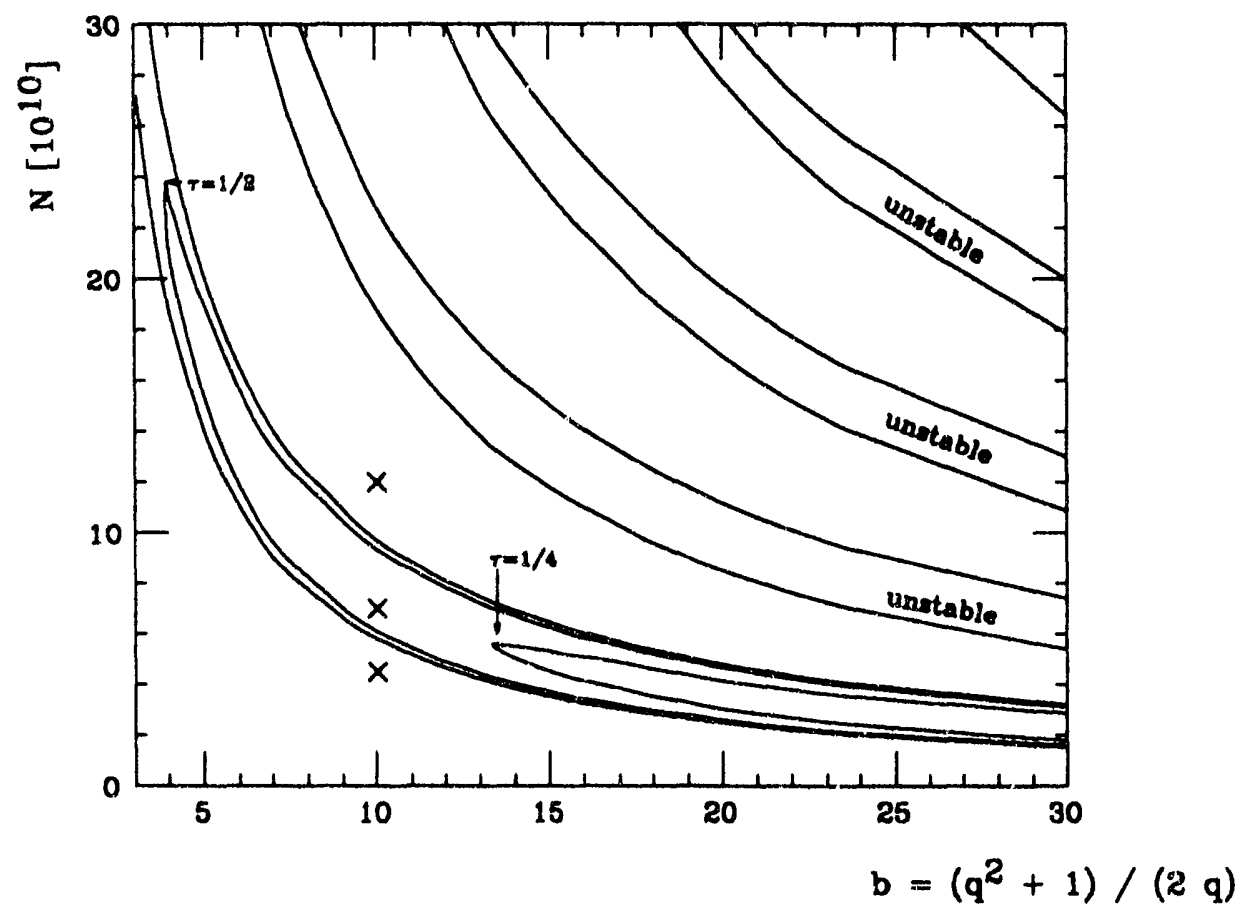

Figure 4. Stable and unstable regions of the linear motion

Next we derive the time evolution of the distribution function. We do this in two steps. First we parameterize the injected beam distribution with the new canonical variables. Then we obtain the time evolution using Hamilton's equation.

We insert the new canonical variables given by Eq. 16 into Eq. 9 to obtain

$$
\Psi(I, \Phi, t)=\frac{1}{2 \pi \sigma} \exp \left\{-\frac{I}{\sigma}\left(b_{1}(t)+c_{1}(t) \cos (2 \Phi+2 \theta(t))\right)\right\},
$$

where $b_{1}, c_{1}$ and $\theta$ are related to $b$ and $c$ from Eq. 10 by:

$$
\begin{gathered}
b_{1}=\frac{1}{2}\left[w^{2}(b+c)+\left(\dot{w}^{2}+\frac{1}{w^{2}}\right)(b-c)\right], \quad e_{1}=\frac{1}{2}\left[w^{2}(b+c)+\left(\dot{w}^{2}-\frac{1}{w^{2}}\right)(b-c)\right], \quad d_{1}=-\frac{\dot{w}}{w}(b-c) \\
c_{1}=\sqrt{e_{1}^{2}+d_{1}^{2}}, \quad \tan (2 \theta)=-\frac{d_{1}}{e_{1}} .
\end{gathered}
$$


At injection $t=0$ we have

$$
\Psi(I, \Phi, 0)=\frac{1}{2 \pi \sigma} \exp \left\{-\frac{I}{\sigma}\left(b_{1}(0)+c_{1}(0) \cos (2 \Phi+2 \theta(0))\right)\right\}
$$

From the Hamiltonian (Eq. 18) in action angle variables we have

$$
H=\omega_{s} \frac{I}{w^{2}}
$$

and Hamilton's equation we derive the time evolution of the canonical variables: $I^{\prime}=0, \Phi^{\prime}=\omega_{s} / w^{2}$. Since the time evolution of the distribution function has to undo the motion of the canonical variables we have to replace $\Phi$ by $\Phi-\omega_{s} \int d t / w^{2}$ to obtain

$$
\Psi(I, \Phi, t)=\frac{1}{2 \pi \sigma} \exp \left\{-\frac{I}{\sigma}\left(b_{1}+c_{1} \cos \left(2 \Phi-2 \omega_{s} \int_{0}^{t} \frac{1}{w^{2}} d t+2 \theta\right)\right)\right\}
$$

where $c_{2}, b_{1}$ and $\theta$ stand for $c_{2}(0), b_{1}(0)$ and $\theta(0)$. The distribution function given by Eq. 20 fulfills Liouville's Equation 8:

$$
\frac{\partial \Psi}{\partial t}+\frac{\omega_{s}}{w^{2}} \frac{\partial \Psi}{\partial \Phi}=0
$$

We should focus our attention on the condition $c_{2}=0$ which implies that the distribution function looses its explicit time dependence. We might think about filamentation as a process which is triggered by a beam not matched to the lattice, i.e. the contours of the injected beam distribution are not circles centered on the origin of phase space. It follows that the distribution function which described this non-circular injected beam, must have an explicit time dependence.

In the transverse case, when a beam is injected into a periodic structure, $\partial \Psi / \partial t=0$ is equivalent to a beta-mismatch parameter of unity: $\beta_{m a g}=1$ [9], [1]. With the definitions of $b$ and $c$ in Eq. 10 we obtain (for $\left.c_{2}=0\right)$

$$
w_{0}^{4}+2\left(w_{0}^{2} \dot{w}_{0}^{2}-1\right) q^{4}+\left(\dot{w}_{0}^{2}+\frac{1}{w_{0}^{2}}\right) q^{4}=0 .
$$

This equation clearly admits the trivial solution $q=1, w=1, \dot{w}=0$. It is not clear if a solution exists for $q \neq 1$ since $w$ and $\dot{w}$ are given implicitly by the one-turn map $M$, which depends implicitly on $q$.

\section{Averaging of the Nonlinear Wakeforce}

In Section 2 we attempted to quantify the effectiveness of ergodization. We found "fast" ergodization if we split the wakefield contribution of the Hamiltonian into a linear and a nonlinear part. Here it is more convenient to work with an explicit time-dependent Hamiltonian. We replace $\left(\Phi_{2}=\omega_{s} t, \Phi_{1}=\Phi\right)$ in Eq. 18 and obtain

$$
H(I, \Phi, t)=\omega_{s} \frac{I}{w^{2}}+h_{n w} \quad, \quad h_{n w}=\frac{\omega_{s} \epsilon}{\sqrt{\kappa}}\left(\exp \left(-\frac{I w^{2} \cos (\Phi)^{2}}{\sigma \kappa}\right)+\frac{I w^{2} \cos (\Phi)^{2}}{\sigma \kappa}\right) .
$$

Now we compare this Hamiltonian with an averaged Hamiltonian

$$
H(J, t)^{a}=\omega_{s} \frac{J}{w^{2}}+h_{n w}^{a} \quad, \quad h_{n w}^{a}=\left\langle h_{n w}\right\rangle_{\Phi}=\frac{\omega_{s} \epsilon}{\sqrt{\kappa}}\left(e^{-z} I_{0}(z)+z\right) \quad \text { with } \quad z=\frac{J w^{2}}{2 \sigma \kappa} .
$$

where $I_{0}(z)$ denotes the modified Bessel function. The frequency now depends on the amplitude:

$$
\frac{\partial h_{n w}^{a}}{\partial J}=\frac{\omega_{s}}{w^{2}}+\frac{\omega_{s} \epsilon w^{2}}{2 \sigma \sqrt{\kappa^{3}}}\left(e^{-z}\left(I_{1}(z)-I_{0}(z)\right)+1\right)
$$


We claim that the Hamiltonian in Eq. 23 is a good approximation of Eq. 22 and support this claim by the results achieved in Section 2 and a theorem (by Nekhoroshev [6]), which shows that the difference between exact and averaged trajectory remains small.

$$
\text { Theorem: } \quad|J(t)-I(t)|<\rho \epsilon^{1 / c} \text { for } t \in\left[0, \tau \epsilon^{-3 / 4} e^{1 /(4 c \epsilon)}\right]
$$

where $\rho, \tau$ are dimensional constants and $c=40$. Thus, the time interval where we might have a good approximation becomes smaller with increasing strength of the perturbation. This theorem assumes $\partial^{2} h_{n w}^{a}(J, t) / \partial J^{2}$ to be definite, either positive or negative, which is fulfilled since $I_{1}^{\prime}(z)-I_{0}^{\prime}(z)-I_{1}(z)+I_{0}(z)>0$. The distribution function which takes into account the nonlinear contribution looks like:

$$
\Psi(J, \phi, t)=\frac{1}{2 \pi \sigma} \exp \left\{-\frac{J}{\sigma}\left(b_{1}+c_{2} \cos \left(2 \phi-2 \int_{0}^{t} \frac{\partial h_{n w}^{a}}{\partial J} d t+2 \theta\right)\right)\right\} .
$$

In Figs. 5 and 6 we evaluated the distribution function for three different currents $\left(N=1,3\right.$ and $\left.6 \times 10^{10}\right)$ and compare the results with multi-particle tracking based on the non-averaged Hamiltonian of Eq. 22. We observe an small phase error between the tracking and analytical result.

In Fig. 5, after 108 revolutions, the amplitude-dependent tuneshift has been visualized by plotting the evolution of the initial axis $z=0, \epsilon=0$. We see good agreement in the deformation of the small amplitude ellipses with increasing numbers of injected particles. In Fig. 6, after 500 revolutions, we observe substantial disagreement between the trarking and analytical results starting at $N=3 \times 10^{10}$ particles. This is related to the time interval in which $\mathscr{I}_{I}(J, t)^{a}$ is a good approximation of $H(I, \Phi, t)$. As expected, this time interval decreases as the strength of the rurbation, i.e. $\mathrm{N}$, increases.

\section{Summary}

We study the effect of an inductive wakefield on the evolution of the distribution function with a simplified Hamiltonian model. Due to an assumed mismatch at injection, ihe bunch length and thus the wakeforce will oscillate. The Hamiltonian depends explicitly on time.

In the linear approximation the beam dynamics has bren treated according to the theory of Hill's equations and is very similar to the transverse betatron moticn. The trace of the one-turn map answers the stability question for the linear motion. The time evolution of the distribution function has been derived. The condition $\partial \Psi / \partial t=0$ leads to a possible driving term of the filamentation process.

Averaging over nonlinear perturbation has been discussed from a general standpoint. This method was applied to the nonlinear part of the wakefield term. The results were discussed and compared to multi-particle tracking.

\section{Acknowledgement}

I want to thank Sam Heifets who influenced this work substantially.

\section{Appendix: Filamentation due to nonlinear RF fields}

In section 1 we derived the Hamiltonian for an inductive wakefield based on the unperturbed longitudinal distribution function. In the presence of nonlinear $\mathrm{RF}$ fields the bunch length oscillations would eventually damp out and the Humiltonian will lose its explicit time dependance.

From Fig. 4a we find a typical growth rate of less than one synchrotron oscillation in the unstable regions of the linear equation 15. We look now at a time constant associated with the filamentation process due to nonlinear RF fields. In Ref. [1] the second moment of the distribution function

$$
\Psi(J, \phi, t)=\frac{1}{2 \pi \sigma} \exp \left\{-\frac{J}{\sigma}\left(b+c \cos \left(2 \phi-2 \omega_{s} t+2 \omega_{s} t \mu J\right)\right)\right\}
$$


has been derived as

$$
\left\langle z^{2}\right\rangle=\sigma_{z 0}^{2}\left\{b+\frac{c}{2}\left(Z(t)^{3 / 2} e^{2 i \omega_{0} t}+c . c .\right)\right\} \quad \text { with: } Z(t)=\frac{1}{1-4 \theta^{2}-2 i b \theta}
$$

where $\theta=\mu \sigma_{z 0} \sigma_{e 0} \omega_{s} t$ is linearly increasing with time, $\mu=\frac{h^{2} \alpha}{8 R \nu_{s}}$ characterizes the nonlinear RF field and c.c. stands for the complex conjugate of the preceding term. In the limit $\mu=0 \mathrm{Eq} .25$ becomes the distribution function in Eq. 9 and $\mu$ is related to the averaged part of the Hamiltonian in Eq. 14. We transform $Z(t)$ into polar coordinates

$$
Z(t)=r e^{i \phi} \text { with: } r=\frac{1}{\sqrt{\left(1-4 \theta^{2}\right)^{2}+4 b^{2} \theta^{2}}} .
$$

We are mostly interested in the oscillating part of Eq. 26 which provides the explicit time dependence in the wakefield contribution. We split the second moment into a d.c. and an oscillating part

$$
\left\langle z^{2}\right\rangle=\left\langle\overline{z^{2}}\right\rangle+\left\langle\tilde{z^{2}}\right\rangle \text {. }
$$

Now we obtain for the amplitude of the oscillating part

$$
\left\langle\tilde{z^{2}}\right\rangle_{t}=r^{3 / 2}\left\langle\tilde{z^{2}}\right\rangle_{t=0}
$$

We want to know the time it takes until the amplitude of $\left\langle\tilde{z^{2}}\right\rangle$ decrease by a factor of 2 : From $r^{3 / 2}=1 / 2$ and Eq. 27 we obtain

$$
\theta^{2}=\frac{8-4 b^{2}+\sqrt{\left(8-4 b^{2}\right)^{2}-128+2^{4 / 3} 128}}{32} .
$$

For a strong mismatched beam $b>>1$ we may simplify this relation to the "thumb rules"

$$
\begin{array}{ll}
\text { for } q<<1 & t_{1 / 2}=2 \sigma_{e \infty} \sqrt{2^{4 / 3}-1} /\left(\sqrt{2} \sigma_{z \infty} \sigma_{e 0}^{2} \mu \omega_{s}\right), \\
\text { for } q>>1 & t_{1 / 2}=2 \sigma_{z \infty} \sqrt{2^{4 / 3}-1} /\left(\sqrt{2} \sigma_{e \infty} \sigma_{z 0}^{2} \mu \omega_{s}\right) .
\end{array}
$$

It turns out the time constant associated with the filamentation process does not depend on the minor axis of the injected ellipse. This makes sense since the nonlinear RF fields affect particles of large amplitude. A typical number for the SLC damping rings is $t_{1 / 2} \approx 500$ turns of about 6 synchrotron periods. Thus the growth rate in the unstable regions of Fig. $4 \mathrm{a}$ is stronger than the damping rate which originates from the filamentation process due to the nonlinear RF field.

\section{References}

[1] H. Moshammer, Transient Response of the Beam after Injection into a Storage Ring, SLAC-PUB 5824, May (1992), to be published in Nucl. Instr. Meth. A.

[2] F. J. Decker et al, Dispersion and betatron Matching into the Linac SLAC-LUB 5484, May (1991).

[3] K. Bane, Bunch lengthening in the SLC Damping Rings. SLAC-PUB 5177, February (1990).

[4] V. I. Arnold, Dynamical Systems III, (Springer Verlag, Berlin, 1988).

[5] V. I. Arnold, Mathematical Methods of Classical Mechanics. (Springer Verlag, New York, 1980).

[6] P. Lochak and C. Meunier, Multiphase Averaging for Classical Systems. (Springer Verlag, New York, 1988).

[7] E. Courant et.al., Stability in Dynamical Systems I. SLAC-PUB-3415, (1984).

[8] E. D. Courant and H. S. Snyder, Theory of the Alternating-Gradient Synchrotron. Ann. of Physics: 3, $1-48$ (1958).

[9] Matt Sands, A Beta Mismatch parameter, SLAC-AP-85, April (1991). 

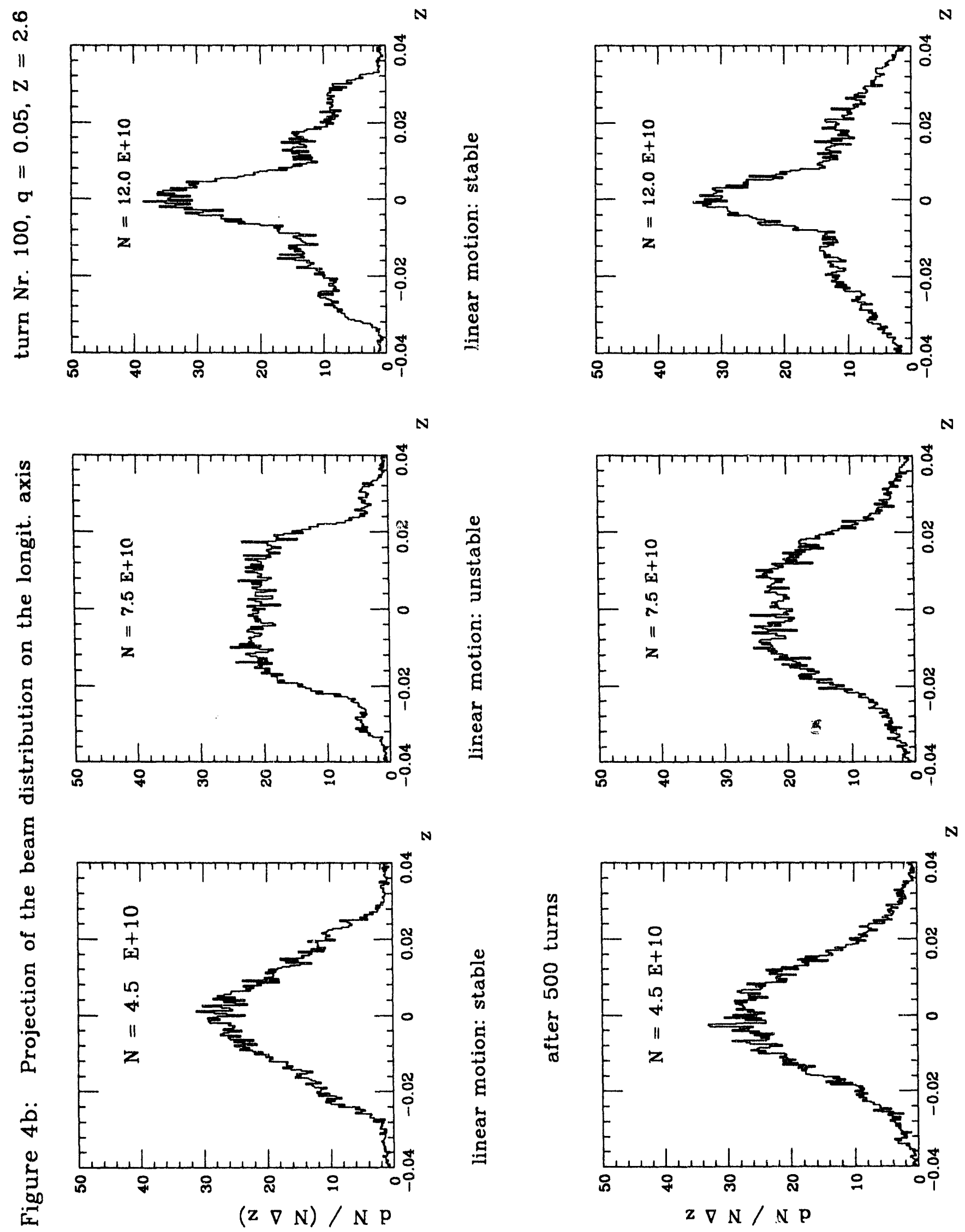

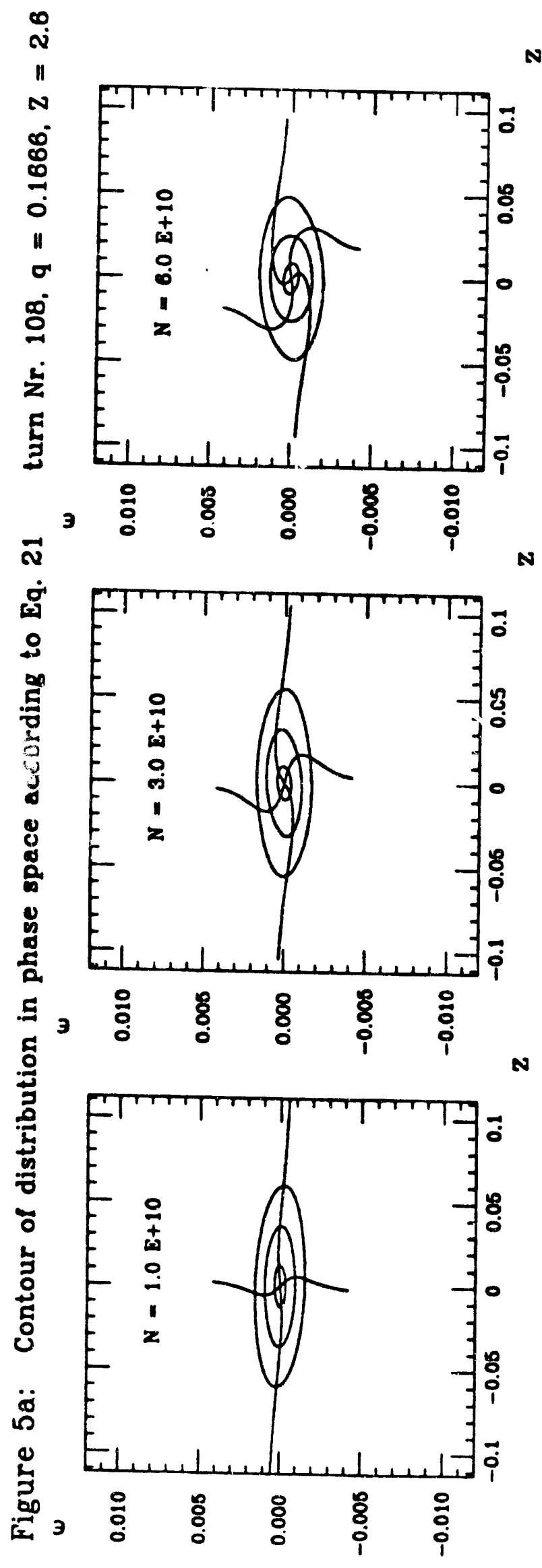

N

$\mathbf{N}$

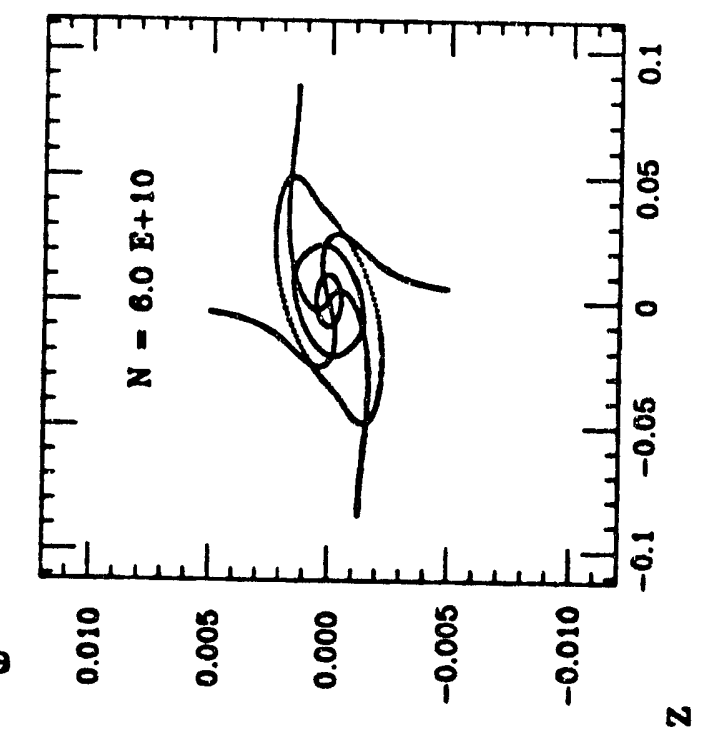

S
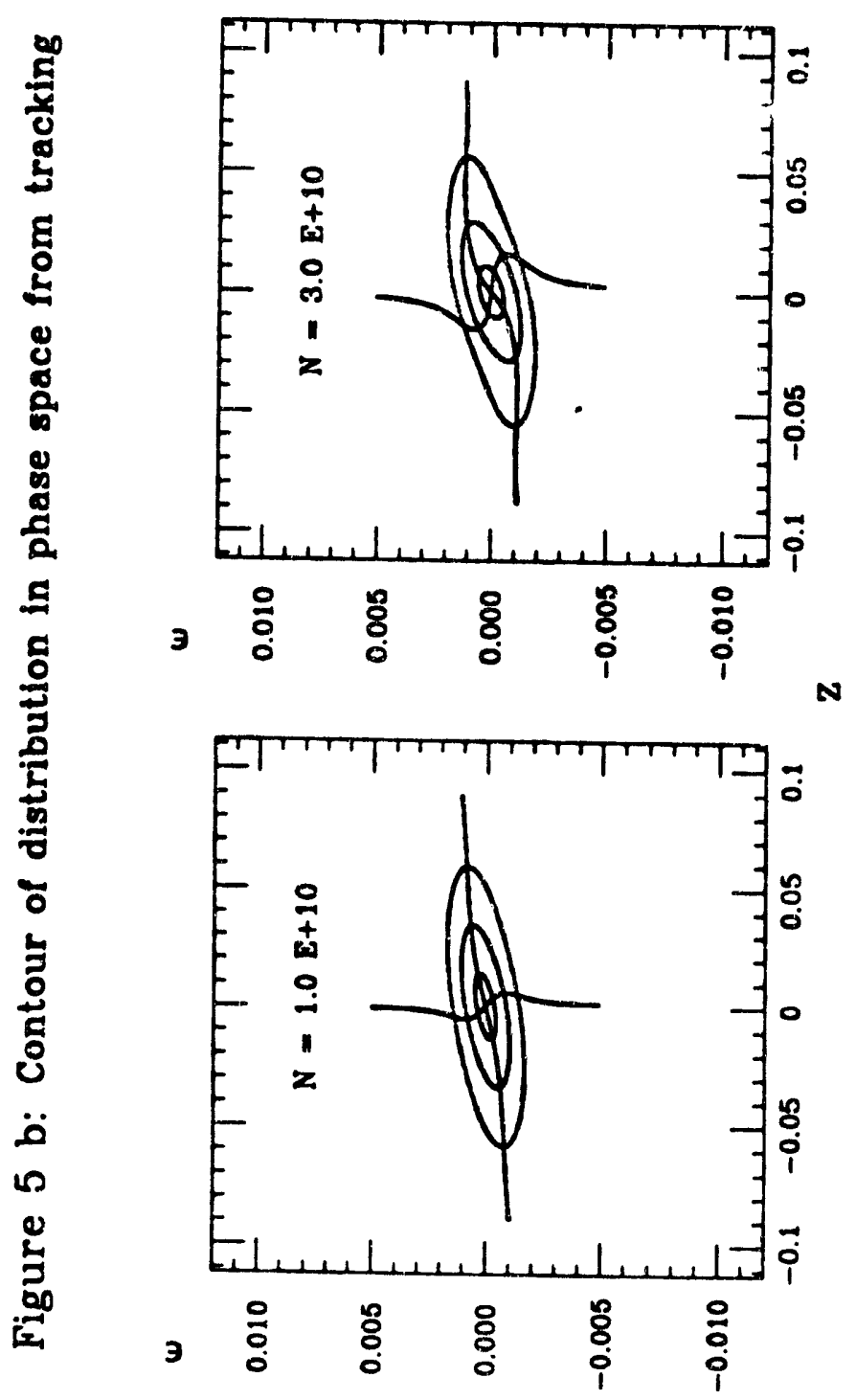


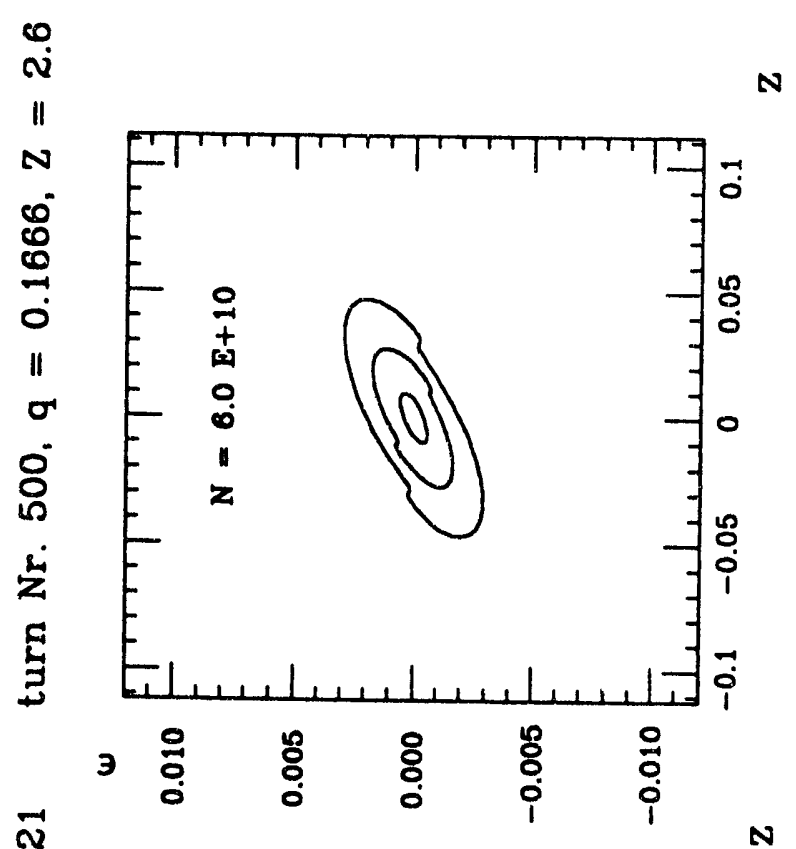

N

N
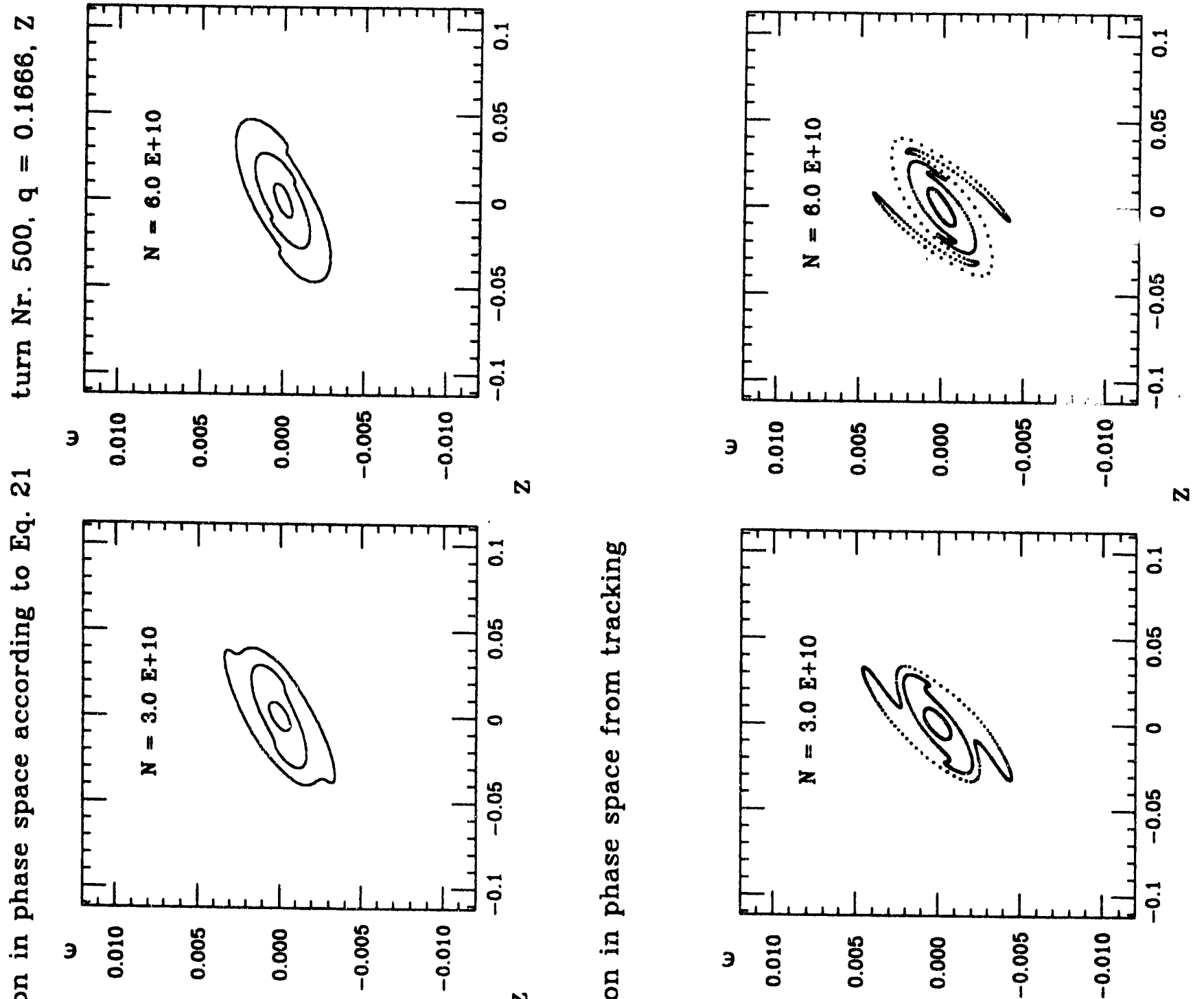

0
0
0
0
0
0
0
0
0
0
0

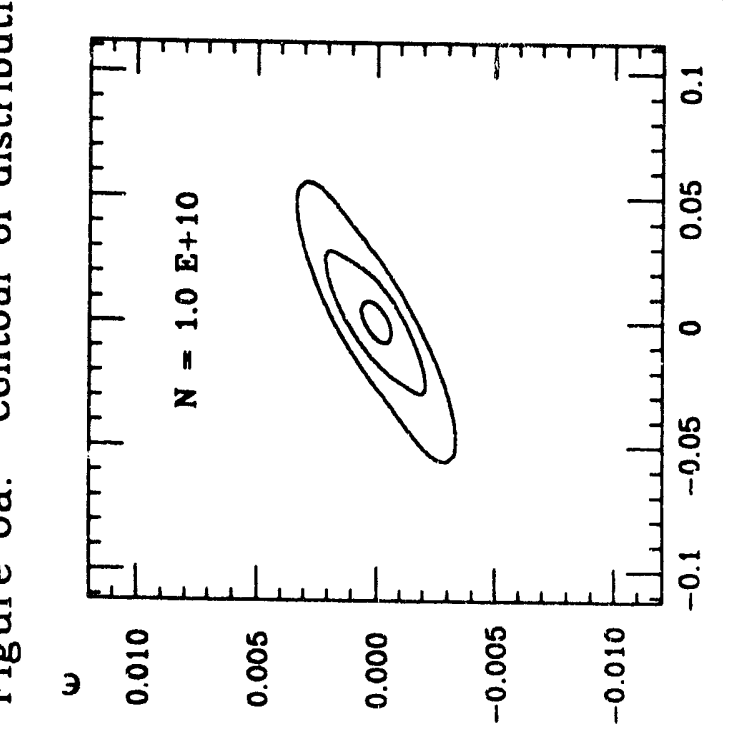

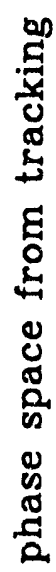

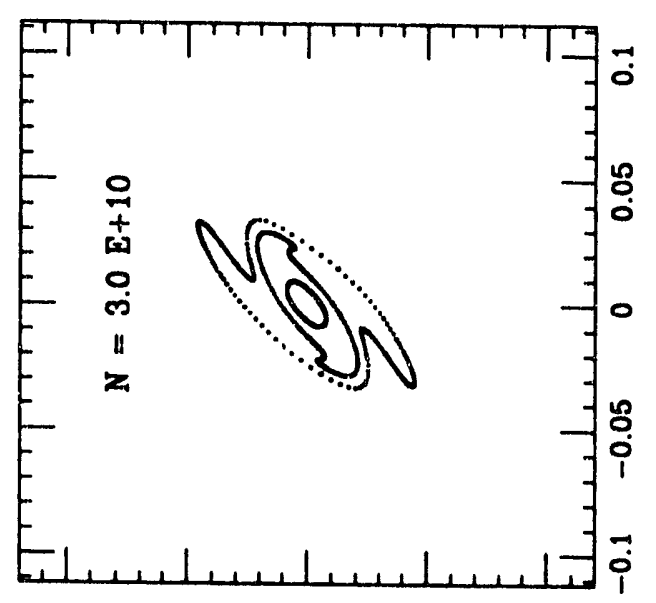

$\begin{array}{llllll}3 & 0 & 0 & 8 & 0 & 0 \\ 0 & 8 & 8 & 0 & 0 \\ 0 & 0 & 0 & 0 & 0\end{array}$

$\mathbf{N}$

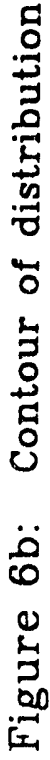

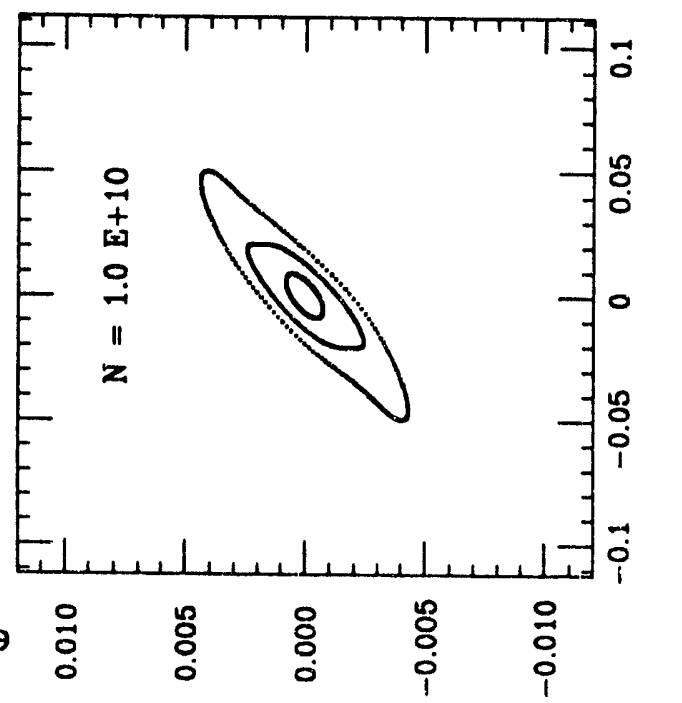



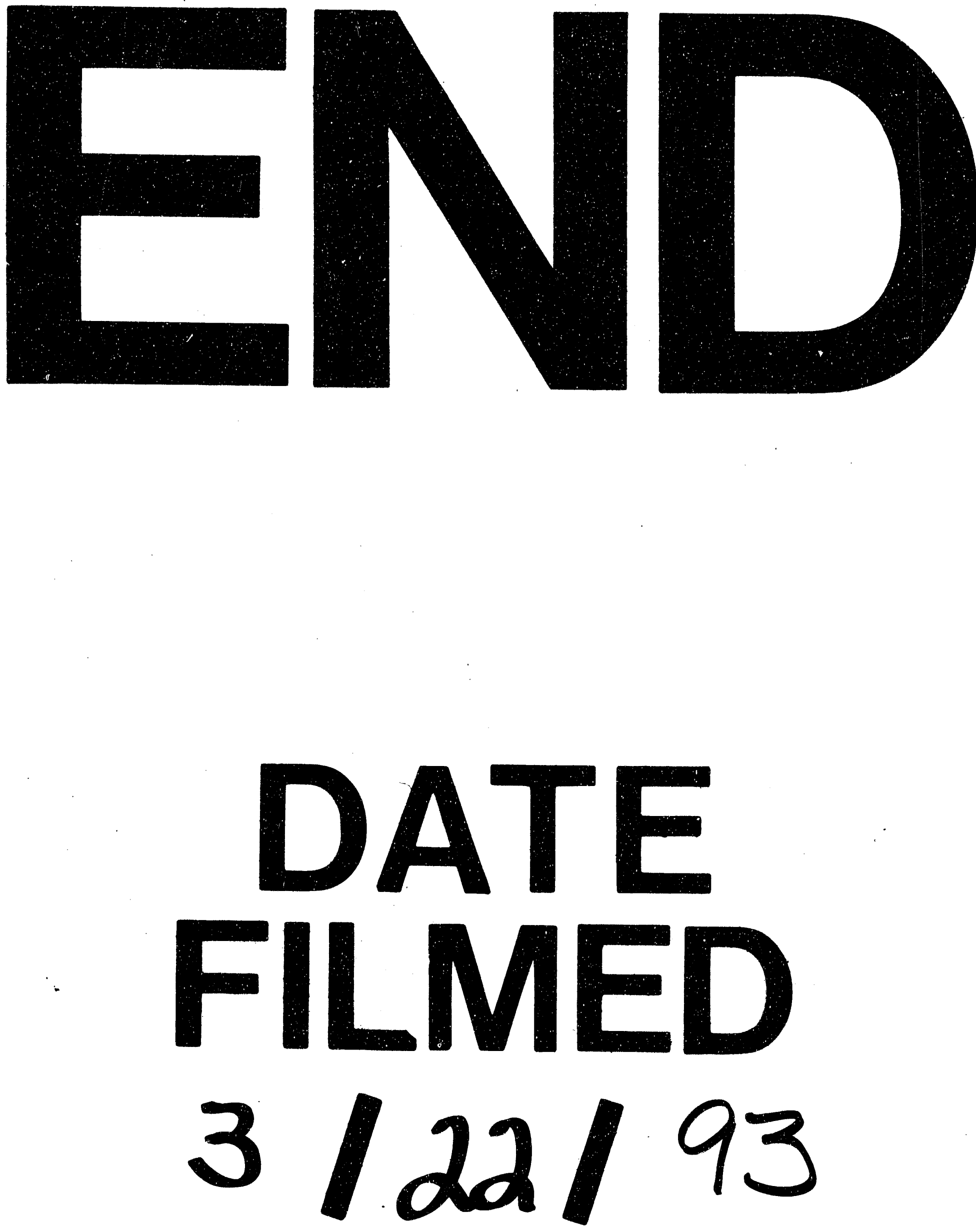
